\title{
Quark Deconfinement inside Compact Stars and Gamma Ray Bursts Inner Engine
}

\author{
Alessandro Drago, Giuseppe Pagliara and Irene Parenti ${ }^{a}$ \\ ${ }^{a}$ Dipartimento di Fisica - Università di Ferrara and INFN Sez. di Ferrara \\ Via Saragat, 1 - 44100 Ferrara - Italy \\ email: drago@fe.infn.it
}

The temporal structure of Gamma Ray Bursts can be interpreted assuming as a inner engine a neutron star which undergoes a progressive compactification via production of strangeness (hyperons and kaons) and quarks. We will propose a tentative identification of various emission periods of the burst with specific structural changes of the star. Each of these modifications of the composition of the compact star takes place as a deflagration and not as a detonation, so the energy released in the transition goes mainly into heat and not into a mechanical wave. This is important in order to avoid an excessive baryonic contamination of the region surrounding the compact star. In this way a ultrarelativistic plasma of electron-positron pairs and of photons can be obtained, powering the Gamma Ray Burst.

\section{Introduction}

In last years the data collected by various X-ray satellites and notably by Beppo-SAX and by Swift have clarified many crucial features of the Gamma-Ray Bursts (GRBs) so that a rather precise phenomenological description of the explosion can now be drawn. The light curve can be separated roughly in four emission periods, although some of these features can often be absent in a specific burst (for a recent review see e.g. [1]).

1) Several bursts present a precursor, namely a small signal containing only a tiny fraction of the total energy of the burst, which anticipates the main event by tens or even hundreds of seconds. The exact fraction of GRBs showing a clear evidence of a precursor is difficult to estimate due to the smallness of the signal. It has been speculated by Lazzati [2] that at least $20 \%$ of the GRBs display a precursor and this fraction could still be an underestimate due to the difficulty of detecting precursors anticipating the main event by only a few seconds. The duration of the precursor is typically of a few seconds.

2) The main event corresponds to the emission with the highest luminosity and is present in the highest energy band of the emission spectrum. The duration of the main event can vary from few seconds (here we are discussing only long bursts, having durations longer than roughly $2 \mathrm{~s}$ ) up to hundreds of seconds. As we will show, it is possible to divide the main event into active periods whose duration can be related to the activity of the so-called inner engine, which is the source of the energy of the burst. The active periods are separated by quiescent times. The high energy photons emitted during the main event 


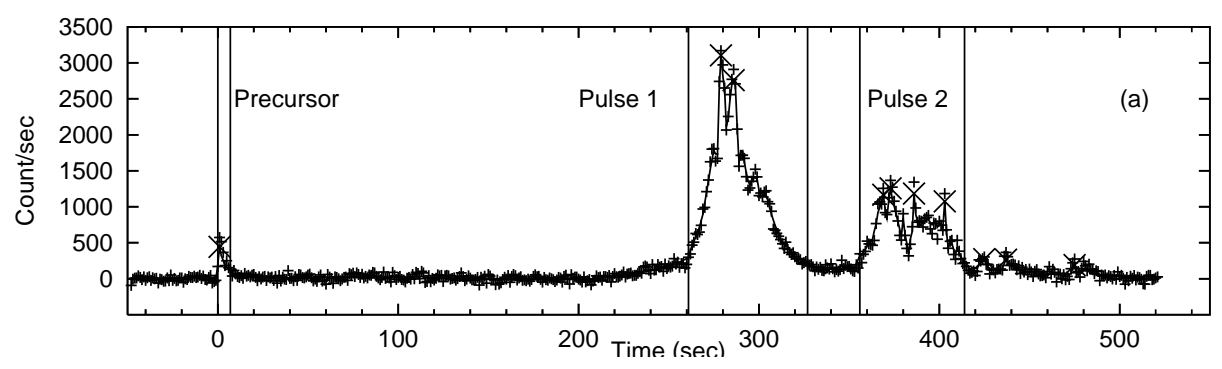

Figure 1. Example of light curve of a GRB. A precursor anticipating the main event by $260 \mathrm{~s}$ is visible. The main emission is divided in two active periods separated by a quiescent time lasting $\sim 30 \mathrm{~s}$. From [3].

are probably associated with internal shocks of the ultrarelativistic jet produced by the inner engine. In this scenario, shells with different velocities collide among themselves producing the detected signal.

3) Swift satellite has recently provided a strong indication that a large fraction of GRBs, after the main event and an initial drop in luminosity, displays a plateau in which the luminosity drops much less rapidly. Inside the plateau some flares can also be present. The luminosity of the plateau is much smaller than that of the main event, but its duration can be much longer, order of thousands of seconds, so that the total energy released can be comparable to the energy released during the main event. The plateau can be originated by a residual activity of the inner engine, although other interpretations are possible.

4) At last the luminosity drops steadily and the so-called afterglow begins. This final prolongated emission is explained in terms of a progressive expansion and slow-down of the ultrarelativistic jet, which interacts with the interstellar medium emitting photons. This last part of the GRB emission is now the less controversial.

In this contribution we concentrate on the main event and we provide some suggestions concerning the precursor.

\section{Quiescent times}

In Fig. 1 1we show an example of GRB in which precursor and quiescent times are clearly visible. The origin of the quiescent times is still debated. Nakar and Piran [4] suggested on a statistical basis that the time intervals during which the GRB shows no activity have a different origin than the time intervals separating peaks within an active period. Fig. 22 clearly indicates that the number of long quiescent times exceeds a stochastic lognormal distribution.

We have recently investigated the structure of the pre-quiescent and of the postquiescent emission [ [5], showing that they share the same micro-structure (see Fig. [3) and also the same emission power and spectral index. Therefore both emissions are generated by a same mechanism which repeats after a quiescent time. It is therefore rather natural to interpret this result as due to different activity periods of the inner engine, during which most of the energy is injected into the fireball. These active periods are 


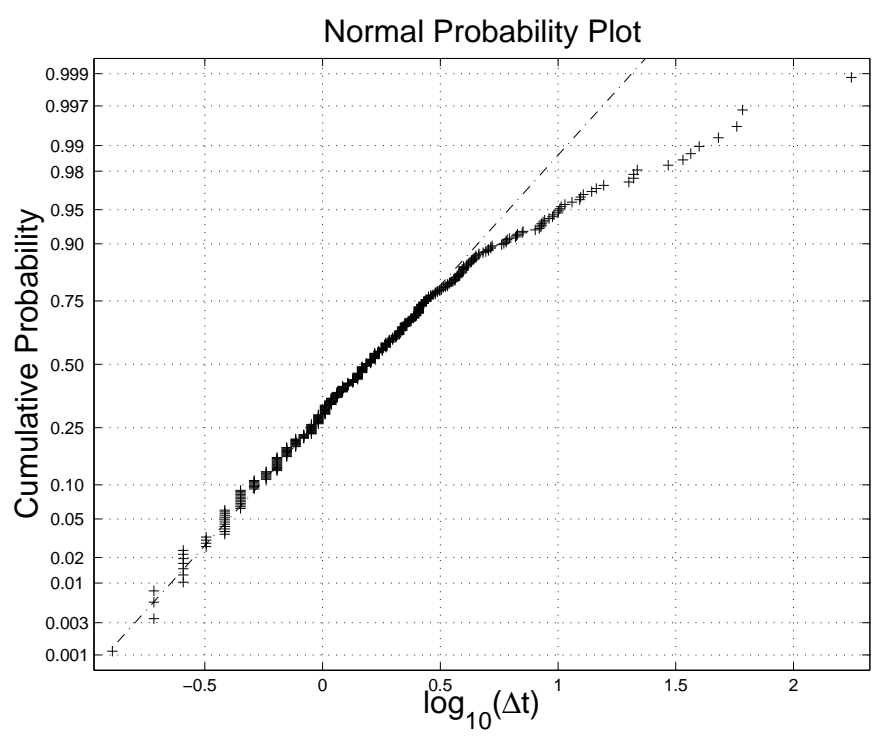

Figure 2. Cumulative probability distribution of the time intervals $\Delta t$ between pulses, compared to a best-fit log-normal distribution. From [4].

separated by quiescent times during which the inner engine remains dormant. The advantage of this interpretation is that it reduces the energy request on the inner engine, the alternative interpretation being that the inner engine remains active and injects energy also during the quiescent times. Moreover, in the latter scenario special conditions on the shells velocity have to be imposed in order to explain why the emission is strongly suppressed although the inner engine remains active. It is possible to show that all GRBs of the BATSE catalogue can be explained by assuming two active periods (which in many cases merge and are therefore not distinguishable). After taking into account the cosmological correction on time intervals $t \rightarrow t /(1+z)$ with $z \sim 2$ for BATSE, the duration of each active period does not exceed $\sim 30 \mathrm{~s}$. In Fig. 4 we show an example of GRB where the duration of the active periods is significantly smaller than the total time interval between the beginning and the end of the main event.

\section{Hadrons to quarks conversion: detonation or deflagration?}

It has been proposed several times that the transition from a star containing only hadrons to a star composed, at least in part, of deconfined quarks can release enough energy to power a GRB [ [6, 17, 8, 9, 10. A crucial question concerns the way in which the conversion takes place, either via a detonation or a deflagration. It has been shown in the past that the mechanical wave associated to a detonation would expel a relatively large amount of baryon from the star surface [11]. In the case of a detonation the region in which the electron-photon plasma forms (via neutrino-antineutrino annihilation near the surface of the compact star) would be contaminated by the baryonic load and it would be impossible to accelerate the plasma up to the enormous Lorentz factors needed to explain 


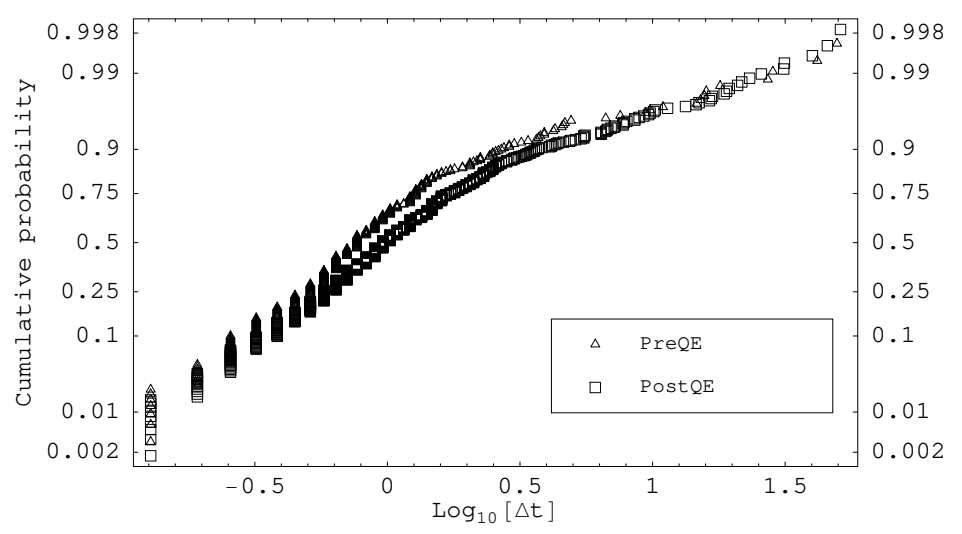

Figure 3. Cumulative probability distribution of the time intervals within the PreQuiescent and the PostQuiescent Emission. The two distributions have a high probability to be equal.

the GRBs.

We have shown in a recent paper [12] that the process of conversion always takes place through a deflagration and not a detonation. In principle the problem of classifying the conversion process can be solved by comparing the velocity of the conversion front to the velocity of sound in the unburned phase. If the velocity of the front it subsonic the process is a deflagration. The velocity of conversion can be estimated in first approximation through energy-momentum and baryon flux conservations through the front. In Fig. 5 we show the result of such a calculation, indicating that the conversion goes through a deflagration with an unstable front. The instability of the front can be deduced by observing that the velocity of sound in the burned phase is smaller than the velocity of that phase in the front frame. The temperature released in the conversion can also be estimated using the thermodynamics first principle.

The problem of computing the actual conversion velocity is anyway more complicated due to fluidodynamical and convective instabilities. Fluidodynamical instabilities are associated with the possibility of the front to form wrinkles. In this way the surface area increases and the conversion can accelerate respect to the laminar velocity $v_{\text {lam }}[13]$. In the absence of new dimensional scales between the minimal dimension $l_{\text {min }}$ and the maximal dimension $l_{\text {min }}$ of the wrinkle, the effective velocity is given by the expression

$v_{\text {eff }}=v_{\text {lam }}\left(\frac{l_{\max }}{l_{\min }}\right)^{D-2}$.

Here $D$ is the fractal dimension of the surface of the front and it can be estimated as $D=2+D_{0} \gamma^{2}$, where $D_{0} \sim 0.6$ and $\gamma=1-\rho_{b} / \rho_{u}$. In this analysis a crucial role is played by neutrino trapping which does not allow the system to reach $\beta$-equilibrium on the same timescale of the conversion process. Taking into account this delay of the weak processes, then $\gamma \leq 0.45$ at all densities. The effect of neutrino trapping is displayed in Fig. 6. Our numerical analysis shows that, although the effective velocity can be significantly 


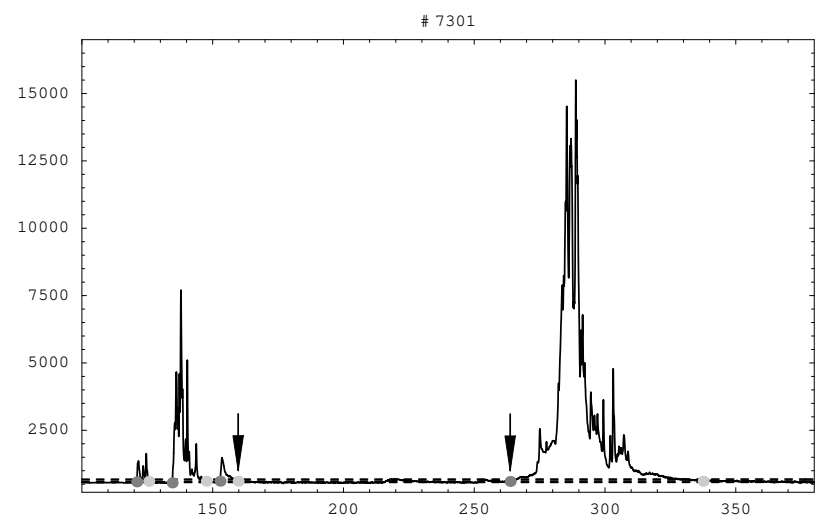

Figure 4. Example of a GRB having a long quiescent time $(\sim 100 \mathrm{~s})$. The durations of the two active periods ( $\sim 40 \mathrm{~s}$ for PreQE and $\sim 80 \mathrm{~s}$ for PostQE) are much shorter than the total duration of the burst $(\sim 220 \mathrm{~s})$.

enhanced respect to the laminar velocity, it is unlikely that $v_{\text {eff }}$ exceeds the speed of sound and therefore the process remains a deflagration.

Convective instability can also instaure, because in a regime of strong deflagration the energy density of the newly formed phase is smaller than the energy density of the old phase. On the other hand, in a high density system in which relativistic corrections are important the new phase forms at a pressure smaller than the pressure of the old phase (here matter is not yet at equilibrium, which is reached only after a delay). Due to this, when the drop of new phase enters the old phase pushed by the gravitational gradient, its pressure rapidly re-equilibrates and its energy density changes accordingly. Quasi-Ledoux convection develops only if the energy density of the new phase remains smaller than that of the old phase after the pressure has equilibrated. In Fig. (7) we show a typical analysis which allows to decide if convection can take place and, in case, which is the convective layer.

Summarizing, the results of our analysis are the followings:

- the conversion always takes place as a strong deflagration and never as a detonation

- fluidodynamical instabilities are present and they significantly increase the conversion velocity but, in realistic cases, the conversion process does not transform from a deflagration to a detonation

- convection can develop in specific cases, in particular it takes place if hyperons are present or if diquark condensate does form.

\section{Structural modifications of the compact star and light curves of the GRBs}

We can combine the information provided in the previous sections and formulate a model for the GRBs based on the following scheme: 


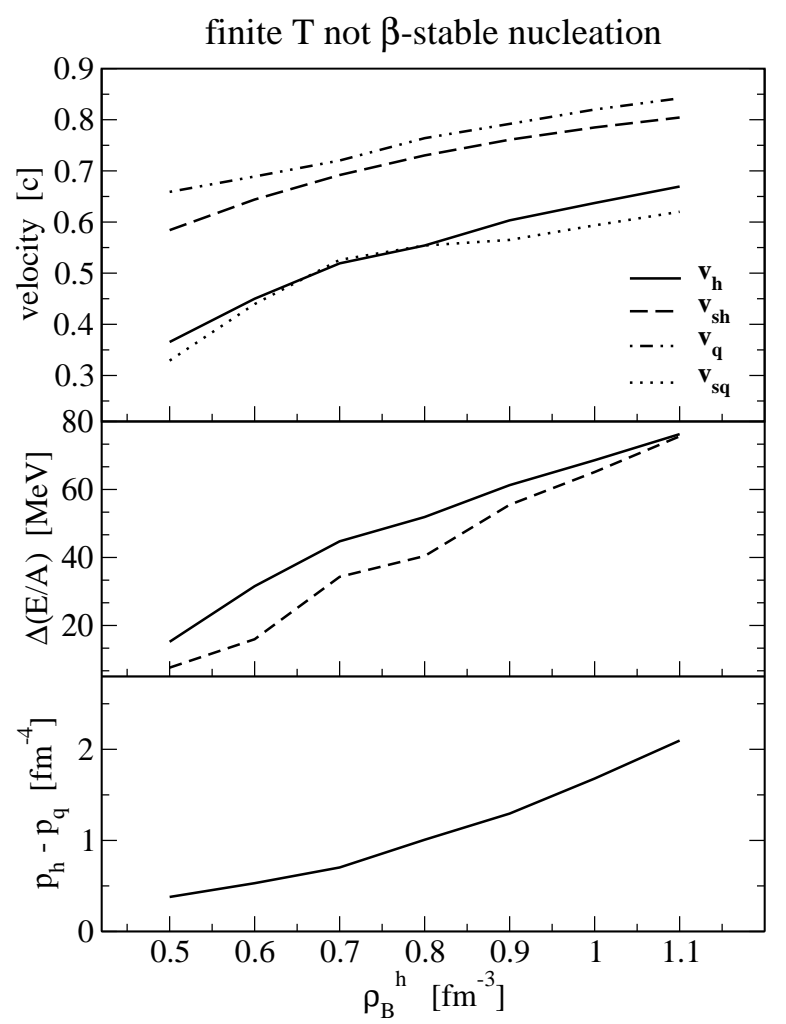

Figure 5. Upper panel: velocity of hadronic phase $v_{h}$, of the burned phase $v_{q}$ and corresponding sound velocities $v_{s h}$ and $v_{s q}$, all in units of the velocity of light and in the front frame. Center panel: energy difference between the two phases (in the hadron phase rest frame). The dashed and the solid lines correspond respectively to the first and to the second iteration in the solution of the fluidodynamics equations. Lower panel: pressure difference between the uncombusted and the combusted phase. Here the combusted phase is obtained using $B^{1 / 4}=170 \mathrm{MeV}$, temperatures from 5 to $40 \mathrm{MeV}$ (as estimated from the solid line in the central panel) and it is not $\beta$-stable.

- a compact star forms after a Supernova explosion. The explosion can be entirely successful or marginally failed, so that in both cases the mass-fallback is moderate (fraction of a solar mass);

- after a delay, varying from seconds to years and dependent on the mass of the compact star and on the mass accretion rate, the star starts readjusting its internal structure. The first event could be associated with the formation of kaon condensation (or of hyperons if it goes through a first order transition [14]). This first structural modification could be relatively small, involving only a modification of the central region of the star, but the presence of strangeness can trigger the instability respect to the formation of strange quark matter. The precursors could be due to this process; 


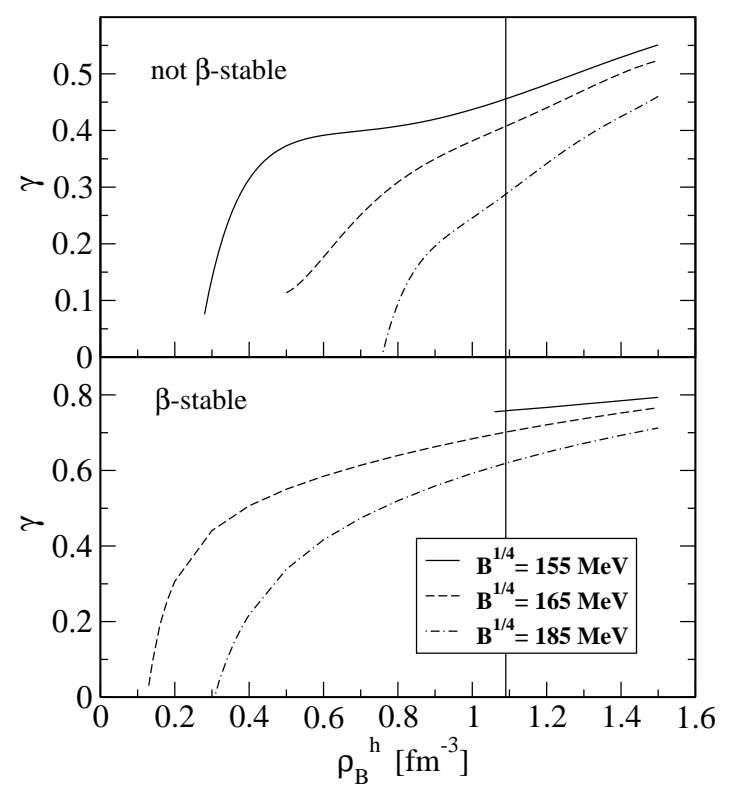

Figure 6. The $\gamma$-factor entering the fractal dimension of the conversion front. See Sec. 3.

- the compact star is now metastable respect to the formation of quark matter (if deconfinement at finite density takes place as a first order transition) and after a short delay the formation of deconfined quarks takes place as a deflagration. A hot compact star remains, and it cools-down through neutrino-antineutrino emission;

- many calculations indicate that Color-Flavor-Locked (CFL) quark matter is the most stable configuration at large density. On the other hand the transition from normal quarks to CFL matter can take place as a first order if the leptonic content of the newly formed normal quark matter phase is not too small [ 15] (its initial leptonic content equals that of the hadronic compact star). In that way, after a short delay (quiescent time) a second transition can take place inside the compact star, due to the formation of superconducting quarks. Energy is again released, and a hot and more compact stellar object is now formed, which again starts cooling via neutrino emission;

- the neutrino-antineutrino emitted by the compact star can annihilate near the surface with an efficiency of order percent. The energy deposited in the electronpositron-gamma plasma can be large enough to power a GRB.

It is interesting to compare the scheme here proposed to the hypernova-collapsar model. In that model the GRB can be associated with a SN explosion which has to be strictly simultaneous with the GRB. In the quark deconfinement model the two events can be temporally separated, with the SN preceding the GRB by a delay which can vary from minutes to years. Arguments in favor of a two-steps mechanism have been discussed in the literature [16]. 


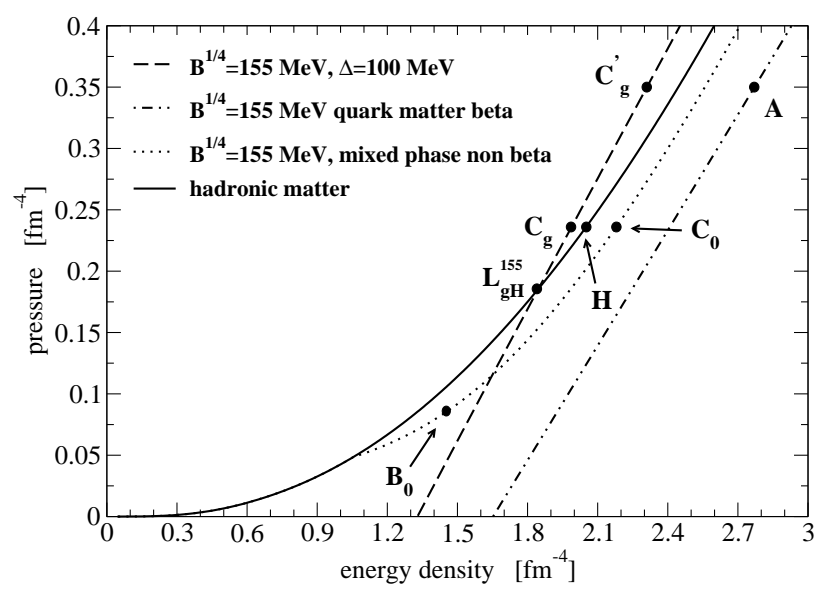

Figure 7. Scheme for convection: $\mathrm{H}$ represents the drop of hadronic matter just before deconfinement, $\mathrm{B}_{0}$ represents the drop of newly formed QM, C stays for the drop of QM after pressure equilibration and $\mathrm{L}$ indicates the end point of the convective layer. Finally A represents a drop of ungapped quark matter before its transition to CFL phase. From $[12$.

We would like to thank Andrea Lavagno for a longstanding collaboration. Most of the results here discussed have been obtained working together.

\section{REFERENCES}

1. P. Meszaros, Rept. Prog. Phys. 69 (2006) 2259.

2. D. Lazzati, Mon. Not. Roy. Astron. Soc. 357 (2005) 722.

3. S. McBreen et al., astro-ph/0604455.

4. E. Nakar and T. Piran, Mon. Not. Roy. Astron. Soc. 331 (2002) 40.

5. A. Drago and G. Pagliara, astro-ph/0512602.

6. K.S. Cheng and Z.G. Dai, Phys. Rev. Lett. 77 (1996) 1210.

7. X.Y. Wang et al., Astron.Astrophys. 357 (2000)543.

8. R. Ouyed and F. Sannino, Astron.Astrophys. 387 (2002) 725.

9. Z. Berezhiani et al., Astrophys.J. 586 (2003) 1250.

10. B. Paczynski and P. Haensel, Mon. Not. Roy. Astron. Soc. 362 (2005) L4.

11. C.L. Fryer and S.E. Woosley, Astrophys.J. 501 (1998) 780.

12. A. Drago, A. Lavagno and I. Parenti, astro-ph/0512652.

13. S.Iv. Blinnikov and P. Sasorov, Phys. Rev. E53 (1996) 4827.

14. J. Schaffner-Bielich et al., Phys. Rev. Lett. 89 (2002) 171101.

15. S.B. Ruster et al., Phys. Rev. D. 73 (2006) 034025.

16. C. Dermer, proceedings of 10th Marcel Grossmann Meeting, Rio de Janeiro 2003, World Scientific, astro-ph/0404608. 